$$
-
$$


THIS PAGE INTENTIONALLY LEFT BLANK 
Published with the assistance of a grant from the Ford Foundation

under its program for the support of publications in the humanities and social sciences 
THIS PAGE INTENTIONALLY LEFT BLANK 


\section{MEXICAN FINANCIAL DEVELOPMENT}

By Dwight S. Brothers, Rice University and Leopoldo Solís M., El Colegio de México

AUSTIN LONDON $\begin{array}{lllllllllllllllllllllll}\text { U } & \text { N } & \text { I } & \text { V } & \text { E } & \text { R } & \text { S } & \text { I } & \text { T } & \text { Y } & \text { O } & \text { F } & \text { T } & \text { E } & X & \text { A } & \text { S } & \text { P } & R & \text { E } & \text { S } & \text { S }\end{array}$ 
The sections of this book originally drafted in Spanish were translated into English by Marian Berdecio.

Library of Congress Catalog Card No. 65-11148

Copyright (c) 1966 by Dwight S. Brothers and Leopoldo Solís M. All Rights Reserved

Printed by The University of Texas Printing Division, Austin Bound by Universal Bookbindery, Inc., San Antonio 